granulocyte-macrophage (GM) colony-stimulating factor (9). Although these autoantibodies have not been reported in patients with Listeria infections, susceptibility to infection caused by this bacterium is increased in GM-colonystimulating factor $-/-$ mice (10). Autoantibodies against GM-colony-stimulating factor or perhaps other cytokines might have impaired the patient's host defense against these organisms; unfortunately, our report is limited by lack of serum for further testing.

This case demonstrates the difficulties of managing patients with $C$. gattii infection and an unusual co-infection with $L$. monocytogenes. Initiation of corticosteroids for the management of severe cryptococcal disease should be undertaken with caution. The differential diagnosis for worsening cryptococcal disease should include acute or subacute bacterial meningitis, particularly when the patient is receiving corticosteroids for the management of immune reconstitution inflammatory syndrome or associated complications.

\section{Acknowledgments}

We thank the patient's family for granting permission to publish this case.

\section{References}

1. Musabende M, Mukabatsinda C, Riviello ED, Ogbuagu O. Concurrent cryptococcal meningitis and disseminated tuberculosis occurring in an immunocompetent male. BMJ Case Rep. 2016. pii:bcr2015213380. http://dx.doi.org/10.1136/bcr-2015-213380

2. Chen SC; Australasian Society for Infectious Diseases (ASID) Mycoses Interest Group. Cryptococcosis in Australasia and the treatment of cryptococcal and other fungal infections with liposomal amphotericin B. J Antimicrob Chemother. 2002;49 Suppl 1:57-61. http://dx.doi.org/10.1093/jac/49.suppl_1.57

3. Harris JR, Lockhart SR, Sondermeyer G, Vugia DJ, Crist MB, D’Angelo MT, et al. Cryptococcus gattii infections in multiple states outside the US Pacific Northwest. Emerg Infect Dis. 2013;19:1620-6.. http://dx.doi.org/10.3201/eid1910.130441

4. Perfect JR, Dismukes WE, Dromer F, Goldman DL, Graybill JR, Hamill RJ, et al. Clinical practice guidelines for the management of cryptococcal disease: 2010 update by the Infectious Diseases Society of America. Clin Infect Dis. 2010;50:291-322. http://dx.doi.org/10.1086/649858

5. Franco-Paredes C, Womack T, Bohlmeyer T, Sellers B, Hays A, Patel K, et al. Management of Cryptococcus gattii meningoencephalitis. Lancet Infect Dis. 2015;15:348-55. http://dx.doi.org/10.1016/S1473-3099(14)70945-4

6. Phillips P, Chapman K, Sharp M, Harrison P, Vortel J, Steiner T, et al. Dexamethasone in Cryptococcus gattii central nervous system infection. Clin Infect Dis. 2009;49:591-5. http://dx.doi.org/10.1086/603554

7. Beardsley J, Wolbers M, Kibengo FM, Ggayi A-BM, Kamali A, Cuc NTK, et al.. Adjunctive dexamethasone in HIV-associated cryptococcal meningitis. N Engl J Med. 2016;374:542-54. http://dx.doi.org/10.1056/NEJMoa1509024

8. Springer DJ, Billmyre RB, Filler EE, Voelz K, Pursall R, Mieczkowski PA, et al. Cryptococcus gattii VGIII isolates causing infections in HIV/AIDS patients in southern California: identification of the local environmental source as arboreal.

PLoS Pathog. 2014; 10:e1004285. http://dx.doi.org/10.1371/ journal.ppat.1004285

9. Saijo T, Chen J, Chen SC-A, Rosen LB, Yi J, Sorrell TC, et al. Anti-granulocyte-macrophage colony-stimulating factor autoantibodies are a risk factor for central nervous system infection by Cryptococcus gattii in otherwise immunocompetent patients. MBio. 2014;5:e00912-4. http://dx.doi.org/10.1128/mBio.00912-14

10. Zhan Y, Lieschke GJ, Grail D, Dunn AR, Cheers C. Essential roles for granulocyte-macrophage colony-stimulating factor (GM-CSF) and G-CSF in the sustained hematopoietic response of Listeria monocytogenes-infected mice. Blood. 1998;91:863-9.

Address for correspondence: Robert G. Deiss, Division of Infectious Diseases, Naval Medical Center of San Diego, 34800 Bob Wilson Dr, San Diego, CA 92134, USA; email: robert.g.deiss.ctr@mail.mil

\title{
Melioidosis in Travelers Returning from Vietnam to France
}

\section{Jérôme Gauthier, ${ }^{1}$ Patrick Gérôme, ${ }^{1}$ Maryline Defez, ${ }^{1}$ Fabienne Neulat-Ripoll, Barbara Foucher, Thierry Vitry, Lionel Crevon, Eric Valade, François M. Thibault, Fabrice V. Biot}

Author affiliations: Hôpital d'Instruction des Armées Desgenettes, Lyon, France (J. Gauthier, P. Gérôme, B. Foucher, T. Vitry, L. Crevon); Centre Hospitalier Universitaire, Grenoble, France (M. Defez); Institut de Recherche Biomédicale des Armées, Brétigny-sur-Orge, France (F. Neulat-Ripoll, E. Valade, F.V. Biot); Ecole du Val-de-Grâce, Paris, France (E. Valade); Direction Centrale du Service de Santé des Armées, Paris (F.M. Thibault)

DOI: http://dx.doi.org/10.3201/eid2209.160169

To the Editor: Melioidosis, a potentially fatal infectious disease, occurs predominantly across much of Asia and in northern Australia because of the soil and water bacterium Burkholderia pseudomallei (1). We report 2 related cases of suppurative cervical lymphadenitis, an unusual adult presentation of melioidosis, in 2 men who returned to France from Vietnam on the same trip (2).

Patient 1, a 28-year-old previously healthy man, was admitted to our hospital in Lyon, France, in October 2013 for the evaluation of a palpable neck mass, which had been growing steadily for the previous 2 months. Examination of the head and neck revealed a fluctuant, tender mass located in the inferior angle of the right side of the mandible, mimicking lymph node tuberculosis. Ultrasonographic investigation confirmed a level II enlarged cervical lymph node

${ }^{1}$ These authors contributed equally to this article. 
that was $2.8 \mathrm{~cm}$ in diameter. The routine bacterial culture from an ultrasound-guided fine needle aspiration showed a microorganism that was identified as $B$. pseudomallei by matrix-assisted laser desorption/ionization time-of-flight mass spectrometry analysis with an extended database. An investigation by local medical staff revealed that the symptoms of our patient began in August 2013, when he returned to France after attending a wedding ceremony in Vietnam, a country to which $B$. pseudomallei is known to be endemic (3). No environmental risk factors, such as the percutaneous inoculation of contaminated material, ingestion, or inhalation, which are the main routes of transmission of melioidosis, were reported (1). The interview of patient 1 identified a co-traveler with similar symptoms (patient 2), who was subsequently admitted to the same hospital.

Patient 2, a 31-year-old previously healthy man, reported a 2-month history of a painful, inflamed, gradually enlarging, right-sided neck mass, accompanied by weight loss, night sweats, and intermittent fevers. Examination showed an enlarged cervical lymph node that was confirmed as level III, $3 \mathrm{~cm}$ in diameter. After noncontributory culture results from an ultrasound-guided fine needle aspiration, we performed an open biopsy under general anesthesia. We excised an adherent, enlarged, pus-filled lymph node and necrotic tissue for microbiologic testing. A real-time PCR assay specifically targeting type-3 secretion system genes (orf11 and BpSCU2) quickly revealed the presence of $B$. pseudomallei DNA, and the diagnosis of melioidosis was confirmed by culture, matrix-assisted laser desorption/ionization time-of-flight mass spectrometry, and antibiogram (4).

For both patients, a cervical, chest, abdominal, and pelvic computed tomographic scan showed no other foci of infection, and a 14-day regimen of intravenous ceftazidime therapy was administered, followed by oral treatment with cotrimoxazole for 6 months (5). However, the neck mass of patient 1 was still swollen after 1 month of treatment, and oral amoxicillin/clavulanic acid was added to cotrimoxazole for 2 months based on an antibiogram from a new bacterial isolation. For patient 2, cotrimoxazole was switched to amoxicillin/clavulanic acid after a presumed adverse drug reaction. At last report, both patients had been disease free for 20 months.

As soon as the second case of melioidosis was confirmed, local and national public health authorities in France were notified, and a larger contact investigation was initiated because 16 other travelers attended the same wedding ceremony before returning to their home countries. None of these travelers had any symptoms of melioidosis. No serologic testing was performed.

Although sporadic cases of travel-associated melioidosis are regularly reported, such case clusters occurring in returning travelers is rare but underscores the role of the contact investigation in this context (6-8). Phylogenetic analyses, performed by a 7-locus multilocus sequence typing analysis, revealed that the 2 isolates shared the same sequence type (381) (identification nos. 4488 and 4489, http:// pubmlst.org/bpseudomallei), which was previously identified in Thailand and Cambodia (9), suggesting a clonal infection from a single-point source. The epidemiologic assessment will be completed by whole-genome sequencing.

Most cases of oropharyngeal melioidosis have been reported in children and were believed to be associated with an oral contamination $(1,3)$. The ingestion of unchlorinated or inefficiently chlorinated water from local residences and hotels has been involved in melioidosis outbreaks and could have been the route of infection for these patients. However, the source of infection might also be linked to a scooter ride taken by both men together around a lake in the vicinity of Hanoi (10).

These patients had no known individual risk factor for melioidosis, such as diabetes, hazardous alcohol use, chronic lung or renal disease, thalassemia, glucocorticoid and other immunosuppressive therapy, or cancer, whereas up to $70 \%$ of patients with travel-associated melioidosis had $\geq 1$ predisposing factor $(1,8)$. However, the percentage of patients with an underlying risk factor dropped to $37.5 \%$ when the data excluded patients who were born in melioidosis-endemic countries or others who had a long-term stay in a melioidosis-endemic country (6). This finding makes us cautious not to repeat making the common assumption about the link between underlying conditions and the risk for melioidosis, especially in regard to conventional tourists traveling in melioidosis-endemic areas.

\section{Acknowledgments}

The authors would like to thank Joel Bozue and Nicholas Vietri from the US Army Medical Research Institute of Infectious Diseases for their critical review of this article. We are also grateful to the public health authorities in France, namely the Agence Régionale de Santé Rhône-Alpes and the Institut de Veille Sanitaire, for supporting the investigation.

This work was supported by the French Ministry of Defense (grant no. PDH-2-NRBC-2-B2-201).

\section{References}

1. Currie BJ. Melioidosis: evolving concepts in epidemiology, pathogenesis, and treatment. Semin Respir Crit Care Med. 2015;36:111-25. http://dx.doi.org/10.1055/s-0034-1398389

2. Chlebicki MP, Tan BH. Six cases of suppurative lymphadenitis caused by Burkholderia pseudomallei infection. Trans R Soc Trop Med Hyg. 2006;100:798-801. http://dx.doi.org/10.1016/j. trstmh.2005.10.004

3. Wiersinga WJ, Currie BJ, Peacock SJ. Melioidosis. N Engl J Med. 2012;367:1035-44. http://dx.doi.org/10.1056/NEJMra1204699

4. Thibault FM, Valade E, Vidal DR. Identification and discrimination of Burkholderia pseudomallei, B. mallei, and 
B. thailandensis by real-time PCR targeting type III secretion system genes. J Clin Microbiol. 2004;42:5871-4.

http://dx.doi.org/10.1128/JCM.42.12.5871-5874.2004

5. Dance D. Treatment and prophylaxis of melioidosis. Int J Antimicrob Agents. 2014;43:310-8. http://dx.doi.org/10.1016/j. ijantimicag.2014.01.005

6. Dan M. Melioidosis in travelers: review of the literature. J Travel Med. 2015;22:410-4. http://dx.doi.org/10.1111/jtm.12236

7. Doker TJ, Sharp TM, Rivera-Garcia B, Perez-Padilla J, Benoit TJ, Ellis EM, et al. Contact investigation of melioidosis cases reveals regional endemicity in Puerto Rico. Clin Infect Dis. 2015; 60:243-50. http://dx.doi.org/10.1093/cid/ciu764

8. Saïdani N, Griffiths K, Million M, Gautret P, Dubourg G, Parola P, et al. Melioidosis as a travel-associated infection: case report and review of the literature. Travel Med Infect Dis. 2015;13:367-81. http://dx.doi.org/10.1016/j.tmaid.2015.08.007

9. McCombie RL, Finkelstein RA, Woods DE. Multilocus sequence typing of historical Burkholderia pseudomallei isolates collected in Southeast Asia from 1964 to 1967 provides insight into the epidemiology of melioidosis. J Clin Microbiol. 2006;44:2951-62. http://dx.doi.org/10.1128/JCM.00725-06

10. Limmathurotsakul D, Wongsuvan G, Aanensen D, Ngamwilai S, Saiprom N, Rongkard P, et al. Melioidosis caused by Burkholderia pseudomallei in drinking water, Thailand, 2012. Emerg Infect Dis. 2014;20:265-8. http://dx.doi.org/10.3201/eid2002.121891

Address for correspondence: Fabrice V. Biot, Institut de Recherche Biomédicale des Armées, Département de Biologie des Agents Transmissibles, Unité de Bactériologie, UMR-MD1, B.P. 73, 91220 Brétigny-sur-Orge, France; email: fabrice.biot@irba.fr

\section{mcr-1-Positive Colistin- Resistant Escherichia coli in Traveler Returning to Canada from China}

\author{
Michael Payne, ${ }^{1}$ Matthew A. Croxen, ${ }^{1}$ \\ Tracy D. Lee, Brian Mayson, Sylvie Champagne, \\ Victor Leung, Sherri Bariso, Linda Hoang, \\ Christopher Lowe
}

Author affiliations: Providence Health Care, Vancouver, British Columbia, Canada (M. Payne, B. Mayson, S. Champagne, V. Leung, S. Bariso, C. Lowe); The University of British Columbia, Vancouver (M. Payne, B. Mayson, S. Champagne, V. Leung, S. Bariso, L. Hoang, C. Lowe); British Columbia Centre for Disease Control Public Health Laboratory, Vancouver (M. Croxen, T.D. Lee, L. Hoang)

DOI: http://dx.doi.org/10.3201/eid2209.160177

To the Editor: A 61-year-old man underwent transurethral prostate resection in Vancouver, British Columbia,

${ }^{1}$ These authors contributed equally to this article. in January 2016. On postoperative day 1 , he was febrile $\left(39.1^{\circ} \mathrm{C}\right)$ and had leukocytosis $\left(12.7 \times 10^{9}\right.$ cells/L). Blood and urine cultures were ordered on postoperative day 2 , and ceftriaxone was started. On postoperative day 3 , urine culture grew Escherichia coli (>100 million CFU/L). Susceptibility testing (VITEK2, bioMérieux, Quebec, Canada) indicated a possible extended-spectrum $\beta$-lactamase producer and showed resistance to ampicillin, cefazolin, ceftriaxone, gentamicin, ciprofloxacin, and trimethoprim/sulfamethoxazole; intermediate resistance to tobramycin; and susceptibility to amoxicillin/clavulanate, piperacillin/tazobactam, ertapenem, meropenem, and nitrofurantoin. Treatment was switched to amoxicillin/clavulanate. The urinary catheter was removed 48 hours later. The patient was discharged on postoperative day 5 and completed 14 days of oral amoxicillin/clavulanate. Blood cultures were negative after 7 days' incubation.

The E. coli cultured from the patient underwent further testing and grew in equal amounts on Columbia ColistinNalidixic acid Agar (CNA) with 5\% sheep blood and Columbia agar with 5\% sheep blood (OXOID, Ontario, Canada). This result was brought to the attention of the hospital's medical microbiologist. A colistin Etest (bioMérieux, Quebec, Canada) showed a MIC of $3 \mu \mathrm{g} / \mathrm{mL}$; EUCAST defines colistin resistance as $>2 \mu \mathrm{g} / \mathrm{mL}$ for Enterobacteriaceae (1).

A real-time PCR to detect the mobile colistin resistance $(m c r-1)$ gene was developed at the Provincial Public Health Laboratory by using primers MCR-1F (5'-CATCGCTCAAAGTATCCAGTGG-3'), MCR-1R (5'-CCATGTAGATAGACACCGTTCTCAC-3'), and probe MCR-1P (5'-Cy5-TGCAGACGCACAGCAATGCCTATGATTAO-3') with TaqMan Fast Advanced Master Mix (Life Technologies, Burlington, Ontario, Canada), on an ABI 7500 FAST thermocycler (Applied Biosystems, Foster City, CA) by using manufacturer's' recommended conditions. The $m c r-1$ gene was confirmed by Sanger sequencing by using previously described oligonucleotides (2). The isolate was also PCR-positive for a $b l a_{\text {CTX-M }}$ gene. The strain was sequenced by using MiSeq (Illumina, Victoria, British Columbia, Canada), and predicted to be sequence type 3944 based on multilocus sequence typing databases (http://github.com/tseemann/mlst; http://mlst. warwick.ac.uk/mlst/dbs/Ecoli) and serotype O159:H4 (3). Sequence type 3944 does not belong to any clonal groups; 1 isolate from Asia is in the MLST database. Abricate (http:// github.com/tseemann/abricate) and PlasmidFinder (4) were used to query the SPAdes-assembled genome (5). Results showed that this isolate carries 3 plasmids that have IncR, IncFIA/HI, and IncI2 replicons. The $m c r-1$ gene was found on a 60,599 -nt contig with the IncI2 replicon; this contig is $\approx 87 \%$ identical to pHNSHP45 (Figure) (2). The bla $a_{\text {CTX-M-27 }}$ gene was found on the same contig as the IncFIA/HI replicon, and no resistance genes were found with the IncR replicon.

At the time of $m c r-1$ detection, the patient and all other patients who shared a hospital room with this patient, 